\title{
YOUTUBE Y EL DESARROLLO DE LA COMPETENCIA MATEMÁTICA. RESULTADOS DE UNA INVESTIGACIÓN CUASIEXPERIMENTAL
}

\author{
Antonia Ramírez García \\ Universidad de Córdoba
}

RESUMEN: La finalidad de este trabajo es presentar los resultados alcanzados en competencia matemática por parte del alumnado de cuarto de educación primaria en un centro educativo que ha participado durante el curso 2008/09 en una investigación cuasiexperimental financiada por la Consejería de Educación de la Junta de Andalucía. Esta investigación tenía como objetivo incrementar la competencia matemática del alumnado -resolución de problemas relacionados con la vida cotidiana, conocimiento de los elementos matemáticos básicos en situaciones reales o simulados (medidas y elementos geométricos), aplicación de algoritmos, seguimiento de cadenas argumentales, etc., a partir del empleo de una metodología basada en grupos de nivel curricular y, así, atender a su diversidad; al mismo tiempo, la incorporación de la Web 2.0 a distintas unidades didácticas implementadas nos ha llevado a determinar factores de influencia en la movilización de dicha competencia, tales como el uso del vídeo digital, decisivo en la comprensión de la geometría dinámica.

ABSTRACT: The aim of this paper is to present the results reached in mathematical competence by students of quarter of primary education in an educational center that has taken part during the course 2008/09 in a quasiexperimental investigation financed by the Commission of Education of the Junta of Andalusia. The objetive of this investigation was to increase the mathematical competence of the students -resolution of problems related to the daily life, knowledge of the mathematical basic elements in situations real or simulated (measurements and geometric elements), application of algorithms, argumentations, etc.-, using a methodology based on groups of level curricular and to attend on his diversity; at the same time, the incorporation of the Web 2.0 to different didactic implemented units has led us to determining factors of influence in the mobilization of mathematical competence, such as the use of the digital, decisive video in the comprehension of the dynamic geometry.

PALABRAS CLAVE: Competencia matemática, Web 2.0., vídeo digital, Youtube. KEYWORDS: Mathematical competence, Web 2.0., digital video, Youtube. 


\section{INTRODUCCIÓN}

Durante el curso 2008/09 se ha Ilevado a cabo en el CEIP Gran Capitán (MontiIla, Córdoba), junto con otros cuatro centros educativos de la capital y la provincia, una investigación cuasiexperimental financiada por la Consejería de Educación de la Junta de Andalucía (PIV-003/08 "Desarrollo de la competencia matemática a través de una metodología basada en grupos de nivel"), como consecuencia de la misma, el grupo de investigación diseñó e implementó tres unidades didácticas -la primera de carácter monográfico sobre resolución de problemas, la segunda centrada en las unidades de medida y la tercera vinculada a la geometría- para comprobar los resultados obtenidos por el alumnado de cuarto de educación primaria en una prueba de evaluación (pretest-postest) tras aplicar una metodología basada en grupos de nivel en el área de matemáticas con el fin de atender a la diversidad del alumnado. La utilización de la Web 2.0 en dichas unidades didácticas ha generado mejoras constatables en el nivel curricular del alumnado en el área de Matemáticas.

El trabajo que presentamos se centra en el centro educativo señalado, puesto que corresponde a un colegio ordinario en el que no se desarrollan planes de bilingüismo, no es un centro TIC, que incorpore las tecnologías de la información y comunicación a las actuaciones docentes, ni DIG, que ofrezca un servicio digital a agentes externos como las familias en aspectos tales como la teletramitación de solicitudes e impresos, avisos, etc., en definitiva, es un centro que representa a la mayor parte de los colegios de educación infantil y primaria de Andalucía. En este centro el uso de las Tecnologías de la Información y la comunicación (TICS) en el aula se circunscribe a situaciones puntuales y aisladas, por lo que su empleo, junto con otras estrategias metodológicas adaptadas al desarrollo de las competencias básicas en la etapa de educación primaria, ha generado cambios positivos en el nivel curricular de partida del alumnado de cuarto tal y como veremos más adelante.

Considerábamos, de acuerdo con Echeverría (1999; 2000) y Navarro (2009) que el uso de las TICS conlleva ir más allá de una "metodología de aula" para adentrarse en un espacio que configure un nuevo sistema educativo fruto de las nuevas necesidades de una generación digital. Sin embargo, Ilegar a esta situación no resulta sencillo, pues hay que romper fuertes reticencias entre los docentes y comenzar con una introducción paulatina que evite miedos y rechazos definitivos hacia las TICS o como señala Castillo (2008) reexaminar qué matemáticas deben aprender los alumnos, así como valorar la mejor forma en que puedan aprenderla en función del nuevo uso que se está haciendo de las TICS. Por su parte, Figueras (2005:8) apunta que en el conjunto de artículos sobre el aprendizaje y la enseñanza de las matemáticas que aparecen en la literatura y en los cuales de forma explícita se menciona el uso de uno o varios de los componentes de las TIC se encuentra una diversidad de estilos y formas de usar estos medios en los procesos educativos que se Ilevan a cabo en la escuela; en este sentido, nos decantamos por el uso de páginas webs y YouTube.

La importancia de algunas páginas web para el desarrollo de la competencia matemática se ha puesto de manifiesto en el análisis Ilevado a cabo por diferentes autores, entre ellos podemos destacar a Cocconi (2008), quien ha realizado la descripción y comparación de tres proyectos extraídos de la Red, dedicados a impulsar el desarrollo y difusión de recursos didácticos virtuales para la movilización de la competencia 
matemática, estos son: NLVM, National Library of Virtual Manipulatives, Utah State University (http://matti.usu.edu/nlvm/nav/); National Council of Teachers of Mathematics, Illuminations (http://illuminations.nctm.org/); y Ministerio de Educación y Ciencia de España, Proyecto Descartes (http://descartes.cnice.mecd.es/). La selección de las páginas utilizadas en la investigación responden a los mismos criterios establecidos por Cocconi (2008) para las páginas anteriores, por San Martín (2003) Godino (2003, 2005) y Franzolin et al. (2006) respecto a otras, así mencionamos como aspectos fundamentales la claridad comunicativa, los aportes originales en el diseño hipermedial y las potencialidades pedagógicas de cada una.

Respecto al vídeo, según las aportaciones de Díaz Arias (2009) hace diez años la World Wide Web se basaba en textos que iban acompañados de fotos y gráficos, mientras que en noviembre de 2008 un 77 por ciento de los internautas norteamericanos visionaron vídeos, estos constituyen un elemento esencial de las interacciones que generan una información globalmente compartida en el ciberespacio (Manovich, 2001:251), a través de un sistema de comunicación propio en el que los usuarios son, dependiendo del caso, emisores o receptores que realizan un intercambio de información acorde con sus gustos y necesidades (Cebrián Herreros, 2008).

A los distintos usos del vídeo en el ciberespacio que establece Díaz Arias (2009:6668) -sociales (entretenimiento, económico e institucional, expresión y construcción de la identidad, redes sociales) e informativos- o Cebrián de la Serna (2005:85-87) -formativa y/o evaluativa para los docentes y estudiantes, informativa, motivadora, expresiva, creativa, lúdica y artística, investigadora de procesos naturales o sociales y comunicativa- podemos añadir una utilización educativa del mismo, la que hemos considerado en esta investigación al emplear la plataforma fundada por Chad Hurley y Steve Chen en 2006, YouTube. Con relación a este uso educativo, Cebrián de la Serna $(2005: 84)$ pone de manifiesto la diferencia existente entre un vídeo didáctico y un vídeo educativo, así los vídeos didácticos suelen llevar su guía y orientación pedagógica concreta, donde nos informan del nivel educativo, áreas o contenidos a tratar (...) los vídeos educativos requieren por nuestra parte un proceso de adaptación al aula, de creación de un proyecto de explotación pedagógico. En nuestra investigación, este último caso ha sido el que se ha utilizado, adaptando la información contenida en el vídeo a la edad, nivel curricular del alumnado, objetivos marcados y contenidos que se pretendían desarrollar. Al mismo tiempo, considerábamos la necesidad de incorporar Youtube como red social con la que se encuentran familiarizados en contextos no educativos y que representa una tendencia a la comunicación interactiva social (Cebrián Herreros, 2008), al tiempo que el sitio web con vídeo online más visitado por adultos (57\%) y jóvenes entre 18 y 24 años (99\%) (Castaño et al. 2008: 99).

Las TICS, como apunta Castillo (2008: 185), pueden apoyar el trabajo del alumnado en varios ámbitos matemáticos como números, medida, geometría, estadística o álgebra; en nuestro caso, han sido la medida y la geometría el objeto de trabajo a través de las TICS -páginas web y Youtube-.

Algunas investigaciones vinculadas a la enseñanza y aprendizaje de las matemáticas y las TICS han demostrado resultados satisfactorios en diferentes etapas educativas; en este sentido, podemos mencionar la investigación desarrollada también en Córdoba, concretamente en el Instituto de Enseñanza Secundaria (IES) Alhaken II 
(Galo y Cañas, 2006) o las Ilevadas a cabo en la Educación Superior de forma genérica para toda la asignatura de Matemáticas (Vílchez Quesada, 2006) y de forma concreta en la Geometría Dinámica (Facello y Osio, 2009).

También podemos hacer referencia al uso de la manipulación de figuras geométricas para crear composiciones de mayor complejidad; en nuestro caso, dicha manipulación partió del visionado del vídeo seleccionado en YouTube y la elección del cuadrado como elemento de referencia para elaborar un hueso nazarí y poder, así, construir un mosaico. Experiencias semejantes han sido las Ilevadas a cabo en el IES Viera y Clavijo en Tenerife utilizando para ello la papiroflexia (Borges Pérez, 2008).

Asimismo, además de la presencia de las TICS, la presente investigación gira en torno a la atención a la diversidad del alumnado; en este sentido, el NCTM (2000) establece que:

Todos los alumnos, independientemente de sus características y circunstancias personales, deben tener oportunidades para estudiar matemáticas y apoyo para aprenderlas. La igualdad no significa que todos deban recibir idéntica instrucción; por el contrario, exige que se hagan adaptaciones razonables y apropiadas para proporcionar la posibilidad a todos los estudiantes de obtener logros.

Esta atención a la diversidad, en nuestro caso, se ha centrado en establecer grupos de nivel -básico, medio y avanzado- para trabajar determinados contenidos matemáticos como veremos más adelante y desarrollar, de este modo, la competencia matemática. El material proporcionado a cada grupo de alumnos y alumnas (cuaderno de trabajo) parte de tareas y/o actividades idénticas, diferenciándose entre ellos por la cantidad y calidad de los apoyos ofrecidos al alumnado; Murillo y Marcos (2007:160) los denominan "ayudas progresivas", éstas pueden consistir en recordar una definición, realizar una sugerencia, proponer un procedimiento, formular una pregunta, aportar un dibujo, etc. Con este sistema de ayudas cada alumno establece su propio itinerario de resolución.

\section{MAterial y metodología}

\subsection{Objetivos}

Los objetivos que se han planteado para esta investigación son los siguientes:

a) Aumentar el nivel de competencia curricular del alumnado de cuarto de educación primaria en el área de Matemáticas.

b) Atender a la diversidad de capacidades, intereses y niveles curriculares del alumnado de un grupo-clase concreto.

c) Desarrollar una metodología de trabajo en el aula a través del establecimiento de grupos de nivel diferenciados (básico, medio y avanzado).

\subsection{Hipótesis de trabajo}

La hipótesis que nos planteamos fue la siguiente: 
1. El alumnado al que se le aplica el programa formativo (grupo experimental) alcanza unos mejores rendimientos académicos en el área de Matemáticas que el alumnado perteneciente al grupo de comparación.

\subsection{Metodología y materiales}

En cuanto a la metodología o como apuntan Arnal, Del Rincón y Latorre la "lógica de la investigación" (1992: 82) seguida en esta investigación, cabe destacar el convencimiento de que el pluralismo metodológico es importante a la hora de resolver cualquier cuestión en nuestro ámbito y que, por tanto, ninguna metodología por sí sola aportará todas las respuestas que pueden realizarse en un determinado contexto educativo. En nuestro caso, como ya aparece formulado en los objetivos, nuestra pretensión es conocer y explicar una realidad -la adquisición de competencias curriculares del área de matemáticas en estudiantes de educación primaria- así como poder llegar a ciertas generalizaciones que puedan predecir comportamientos posteriores en los sujetos objeto del estudio. Son rasgos, por tanto, que definen una metodología empírico-analítica.

El diseño de investigación que se ha desarrollado es de tipo cuasiexperimental, donde se provoca o manipula el fenómeno y determina los valores de las diferentes variables independientes que clasifican a la muestra objeto de estudio y que se ha caracterizado por: el control experimental de la medida, la asignación intencional de los sujetos a los grupos de tratamiento y la no manipulación de las variables independientes.

Dentro de las posibilidades de investigación que ofrece este diseño hemos empleado el modelo pretest-postest con grupo de comparación, en los que se ha efectuado dos medidas de las variables dependientes antes y después de la acción de algún valor de las variables independientes (pretest y postest), proceso implementado en exclusiva en el conjunto experimental. El proceso se ha configurado en cinco grandes fases:

\section{1a Fase. Planificación de la investigación}

La investigación comenzó con un análisis del contexto; seguidamente, se elaboraron las líneas prioritarias del trabajo y se presentó en los centros escolares participantes para su aprobación. Posteriormente, se definió exhaustivamente el problema de investigación, así como los objetivos que han guiado su desarrollo; se operativizaron los objetivos en variables de estudio, se escogió el diseño que ha guiado el trabajo, se describió la muestra objeto de estudio (selección de grupos experimental y de comparación) y seleccionamos y diseñamos los diferentes instrumentos de recogida de información (cuestionarios, pruebas de nivel, escalas de valoración, etc.).

\section{2a Fase. Diseño y desarrollo de las pruebas de control y unidades didácticas}

En un segundo momento, se diseñaron los procedimientos que han servido para recoger toda la información, estos son: prueba de nivel de competencia curricular matemática aplicada en dos momentos (pre y post-test) y que ha servido para dise- 
ñar tres grupos de nivel (básico, medio y avanzado) y valorar la ganancia en competencias; tres unidades didácticas dirigidas a los grupos de nivel y pruebas evaluativas asociadas a las unidades didácticas, en todas ellas se contemplaba la resolución de problemas.

La validez de contenido de la prueba de nivel (pretest-postest) ha sido evidenciada tras la aplicación del método Delphi, idóneo para tratar temas complejos que requieren contrastar y combinar argumentos y opiniones individuales hasta llegar a tomar decisiones no asumibles unilateralmente [(Landeta, 1999) y (Bravo y Arrieta, 2005)]. La constitución del grupo encargado del estudio, definido como grupo monitor, ha estado compuesto por un total de ocho profesionales de las áreas de Didáctica de las Matemáticas pertenecientes a las universidades de Córdoba y Salamanca. Para recoger la información referente a los indicadores del modelo y su definición operativa se enviaron las fichas técnicas elaboradas con una serie de preguntas abiertas donde el grupo tenía libertad de expresión: extensión de las pruebas, redacción y presentación, adecuación de las actividades propuestas para el nivel del alumnado destinatario (cuarto de educación primaria) y observaciones. Una vez recibida esta primera información se evidenció la relevancia que el grupo de expertos había otorgado a cada una de las actividades propuestas y se incorporaron las observaciones planteadas, donde hay que destacar la necesidad de modificar la redacción de alguna de las cuestiones, así como la inclusión de nuevos elementos gráficos que potenciaran el sentido de las mismas. Seguidamente, fue enviado de nuevo al grupo de expertos para un segundo proceso de valoración y consenso. Analizadas las aportaciones ofrecidas por el grupo de expertos en la segunda aplicación del instrumento Delphi, el modelo definitivo apenas sufrió modificaciones y quedó, por lo tanto, constituido por un total de 13 fichas técnicas tendentes a evaluar los conocimientos de matemáticas del alumnado.

Las unidades didácticas diseñadas e implementadas han tomado como referencia tanto la normativa estatal como la autonómica (vid. tabla 1), presentan una serie de contenidos y características propias (vid. tabla 2), asimismo, en cada una de ellas se elaboraron cuadernos de trabajo para los tres niveles curriculares establecidos y sus pruebas de evaluación correspondientes.

Tabla 1. Bloques de contenidos y núcleos temáticos de referencia.

\begin{tabular}{l|l|l}
\hline Unidad Didáctica & Normativa estatal & Normativa autonómica \\
\hline $\begin{array}{l}\text { 1. ¿Quién es Polya? } \\
\text { diciembre. }\end{array}$ & $\begin{array}{l}\text { La resolución de problemas como eje } \\
\text { vertebrador de todos los bloques de } \\
\text { contenidos. }\end{array}$ & $\begin{array}{l}\text { Núcleo temático 1. Resolución de } \\
\text { problemas (transversal). }\end{array}$ \\
\hline $\begin{array}{l}\text { 2. ¿Quiero ser... } \\
\text { arquitect@! }\end{array}$ & $\begin{array}{l}\text { La medida: estimación y cálculo de } \\
\text { magnitudes. }\end{array}$ & $\begin{array}{l}\text { Núcleo temático 4. Desarrollo del } \\
\text { sentido numérico. Medida de } \\
\text { magnitudes. }\end{array}$ \\
\hline $\begin{array}{l}\text { 3. El mosaico de hueso } \\
\text { nazarí. }\end{array}$ & Geometría. & $\begin{array}{l}\text { Núcleo temático 5. Las formas y } \\
\text { figuras y sus propiedades. }\end{array}$ \\
\hline
\end{tabular}

Fuente: Elaboración propia. 
Tabla 2. Contenidos y características que presentan las unidades didácticas implementadas.

\begin{tabular}{|c|c|c|c|}
\hline Número y título & Conocimientos & Destrezas & Actitudes \\
\hline $\begin{array}{l}\text { 1. ¿Quién es } \\
\text { Polya? }\end{array}$ & $\begin{array}{l}\text { 1. La medida del } \\
\text { tiempo: horas y } \\
\text { minutos. } \\
\text { 2. La edad. } \\
\text { 3. El calendario. } \\
\text { 4. El proceso de } \\
\text { compra-venta. } \\
\text { 5. El euro y los } \\
\text { céntimos. } \\
\text { 6. La medición de la } \\
\text { temperatura. }\end{array}$ & $\begin{array}{l}\text { 1. Identificación de los datos } \\
\text { necesarios en una tabla de } \\
\text { información para resolver } \\
\text { problemas de la vida } \\
\text { cotidiana. } \\
\text { 2. Reconocimiento de las } \\
\text { fases de la resolución de } \\
\text { un problema matemático } \\
\text { de acuerdo con la } \\
\text { secuencia establecida por } \\
\text { Polya. } \\
\text { 3. Mecanización de los } \\
\text { algoritmos básicos de la } \\
\text { suma, resta, multiplicación } \\
\text { y división. } \\
\text { 4. Resolución de problemas } \\
\text { relacionados con la vida } \\
\text { cotidiana siguiendo los } \\
\text { pasos establecidos por } \\
\text { Polya. }\end{array}$ & $\begin{array}{l}\text { 1. Interés por } \\
\text { resolver } \\
\text { problemas de } \\
\text { la vida } \\
\text { cotidiana. } \\
\text { 2. Perseverancia } \\
\text { en la } \\
\text { búsqueda de } \\
\text { soluciones } \\
\text { posibles. } \\
\text { 3. Gusto por el } \\
\text { trabajo bien } \\
\text { hecho. }\end{array}$ \\
\hline $\begin{array}{l}\text { 2. ¡Quiero ser... } \\
\text { arquitect@! }\end{array}$ & $\begin{array}{l}\text { 1. La medida de la } \\
\text { longitud. } \\
\text { 2. El metro: } \\
\text { múltiplos y } \\
\text { divisores. } \\
\text { 3. La escala. } \\
\text { 4. El plano y el } \\
\text { mapa. }\end{array}$ & $\begin{array}{l}\text { 1. Medición de objetos de } \\
\text { una habitación. } \\
\text { 2. Elaboración de un plano a } \\
\text { escala de una habitación. } \\
\text { 3. Transformación de } \\
\text { medidas de diferentes } \\
\text { objetos expresadas de } \\
\text { distinto modo. } \\
\text { 4. Expresión de medidas de } \\
\text { forma simple o compleja. } \\
\text { 5. Reconocimiento de las } \\
\text { fases de la resolución de } \\
\text { un problema matemático } \\
\text { de acuerdo con la } \\
\text { secuencia establecida por } \\
\text { Polya. } \\
\text { 6. Mecanización de los } \\
\text { algoritmos básicos de la } \\
\text { suma, resta, multiplicación } \\
\text { y división. } \\
\text { 7. Resolución de problemas } \\
\text { relacionados con la } \\
\text { longitud siguiendo los } \\
\text { pasos establecidos por } \\
\text { Polya. }\end{array}$ & $\begin{array}{l}\text { 1. Interés por } \\
\text { resolver } \\
\text { problemas de } \\
\text { la vida } \\
\text { cotidiana. } \\
\text { 2. Curiosidad por } \\
\text { ampliar } \\
\text { conocimientos } \\
\text { sobre Le } \\
\text { Corbusier. } \\
\text { 3. Perseverancia } \\
\text { en la } \\
\text { búsqueda de } \\
\text { soluciones } \\
\text { posibles. } \\
\text { Gusto por el } \\
\text { trabajo bien } \\
\text { hecho. }\end{array}$ \\
\hline
\end{tabular}




\begin{tabular}{|c|c|c|c|}
\hline $\begin{array}{l}\text { 3. "El mosaico de } \\
\text { hueso nazarí" }\end{array}$ & $\begin{array}{l}\text { 1. Los polígonos: } \\
\text { clasificación y } \\
\text { características. } \\
\text { 2. El perímetro, eje } \\
\text { de simetría y } \\
\text { diagonales. } \\
\text { 3. Figuras simétricas. } \\
\text { 4. El movimiento en } \\
\text { el espacio: } \\
\text { rotación y } \\
\text { traslación. } \\
\text { 5. El área de una } \\
\text { figura. }\end{array}$ & $\begin{array}{l}\text { 1. Elaboración de un hueso } \\
\text { nazarí partiendo de un } \\
\text { cuadrado. } \\
\text { 2. Construcción de un } \\
\text { mosaico decorativo. } \\
\text { 3. Identificación de figuras } \\
\text { geométricas en objetos de } \\
\text { la vida cotidiana. } \\
\text { 4. Trazado del eje de } \\
\text { simetría y diagonales en } \\
\text { aquellas figuras y/o } \\
\text { formas que lo permitan. } \\
\text { 5. Reconocimiento de } \\
\text { figuras simétricas. } \\
\text { 6. Aplicación del concepto } \\
\text { de perímetro, traslación y } \\
\text { rotación. } \\
\text { 7. Reconocimiento del área } \\
\text { de una figura y/o forma. } \\
\text { 8. Reconocimiento de las } \\
\text { fases de la resolución de } \\
\text { un problema matemático } \\
\text { de acuerdo con la } \\
\text { secuencia establecida por } \\
\text { Polya. } \\
\text { 9. Mecanización de los } \\
\text { algoritmos básicos de la } \\
\text { suma, resta, } \\
\text { multiplicación y división. } \\
\text { 10. Resolución de problemas } \\
\text { relacionados con la } \\
\text { geometría siguiendo los } \\
\text { pasos establecidos por } \\
\text { Polya. }\end{array}$ & $\begin{array}{l}\text { 1. Interés por } \\
\text { reconocer } \\
\text { figuras } \\
\text { geométricas en } \\
\text { objetos de la } \\
\text { vida cotidiana. } \\
\text { 2. Curiosidad por } \\
\text { ampliar } \\
\text { conocimientos } \\
\text { sobre la } \\
\text { decoración } \\
\text { islámica. } \\
\text { 3. Perseverancia } \\
\text { en la } \\
\text { búsqueda de } \\
\text { soluciones } \\
\text { posibles. } \\
\text { 4. Gusto por el } \\
\text { trabajo bien } \\
\text { hecho. }\end{array}$ \\
\hline
\end{tabular}

Tabla 2. Contenidos y características que presentan las unidades didácticas implementadas (continuación).

\begin{tabular}{|c|c|c|c|c|c|c|}
\hline Número y título & Temporalización & $\begin{array}{l}\text { Power point } \\
\text { de } \\
\text { presentación }\end{array}$ & Tareas & $\begin{array}{l}\text { Resolución } \\
\text { de } \\
\text { problemas }\end{array}$ & $\begin{array}{l}\text { Actividades } \\
\text { interactivas }\end{array}$ & $\begin{array}{l}\text { Ejercicios } \\
\text { en } \\
\text { cuaderno } \\
\text { de trabajo }\end{array}$ \\
\hline $\begin{array}{l}\text { 1. ¿Quién es } \\
\text { Polya? }\end{array}$ & $\begin{array}{l}10 \text { sesiones } \\
2^{\mathrm{a}} \text { quincena } \\
\text { enero } 2009 \\
\end{array}$ & Sí & No & 5 problemas & No & No \\
\hline $\begin{array}{l}\text { 2. ¡Quiero ser... } \\
\text { arquitect@! }\end{array}$ & $\begin{array}{l}10 \text { sesiones } \\
2^{\underline{a}} \text { quincena de } \\
\text { marzo } 2009\end{array}$ & Sí & 2 & 1 problema & 3 & 7 \\
\hline $\begin{array}{l}\text { 3. "El mosaico de } \\
\text { hueso nazari" }\end{array}$ & $\begin{array}{l}10 \text { sesiones } \\
1^{a \underline{a}} \text { quincena de } \\
\text { mayo } 2009\end{array}$ & Sí & 1 & 1 problema & 5 & 6 \\
\hline
\end{tabular}

Fuente: Elaboración propia. 
Las páginas web utilizadas para trabajar las actividades interactivas quedan expresadas en la tabla 3.

Tabla 3. Páginas web utilizadas.

\begin{tabular}{l|l}
\hline Unidad Didáctica & Páginas web \\
\hline 1. ¿Quién es Polya? & Ninguna \\
\hline 2.iQuiero ser... arquitect@! & $\begin{array}{l}\text { http://redes.agrega.indra.es/repositorio/13062008/es_ } \\
\text { 20080613_3_9161840//mt02_oa04_es/index.html } \\
\text { http://www.isftic.mepsyd.es/w3/recursos/primaria/matematicas/ } \\
\text { longitud/menu.html }\end{array}$ \\
\hline $\begin{array}{l}\text { 3. El mosaico de hueso } \\
\text { nazarí }\end{array}$ & http://www.youtube.com/watch?v=oNnK28Eqmi8\&feature= \\
& PlayList178DB3E63150F322\&playnext=1\&playnext_from= \\
& PL\&index=9 \\
& http://redes.agrega.indra.es/repositorio/13062008/es_ \\
& 20080613_3_9161840//mt02_oa04_es/index.html \\
http://www.isftic.mepsyd.es/w3/recursos/primaria/matematicas/ & superficie/index.html
\end{tabular}

Fuente: Elaboración propia.

De entre estas páginas cabe destacar la dirección del vídeo digital en Youtube, éste constituye el punto de partida y eje central de toda la unidad didáctica, en él se describe e ilustra el proceso de construcción de los mosaicos nazaríes en la Alhambra (Granada). El vídeo se repitió tres veces para que el alumnado pudiera apreciar correctamente la tarea que debía realizar, sobre todo, la secuencia de tiempo comprendida entre los minutos 2:50 y 3:31, en ella se puede observar cómo se transforma un cuadrado en un "hueso nazarí" (vid. figura 1). El vídeo fue visionado por los dos grupos, experimental y de comparación, pero sólo el grupo experimental recibió los apoyos necesarios nivelados en dificultad para ejecutar la tarea que se le solicitaba, la construcción de su propio hueso nazarí y la elaboración de un mosaico con todos los "huesos" realizados por el grupo clase. 
Figura 1. Imagen del hueso nazarí.

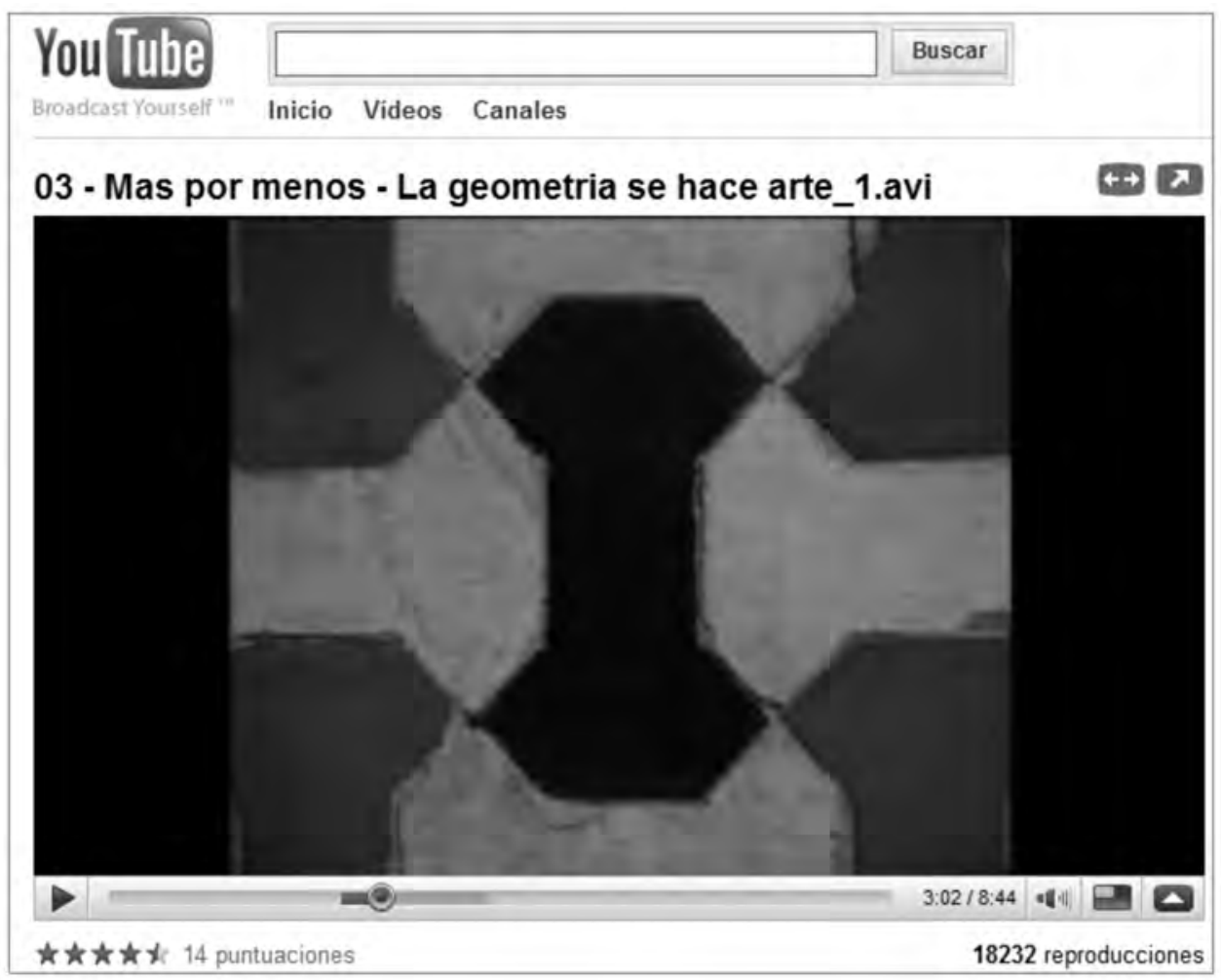

Fuente: http://www.youtube.com/watch?v=oNnK28Eqmj8\&feature=PlayList 178DB3E63150F322\&playnext=1 \&playnext_from=PL\&index=9.

\section{3몰. Aplicación de instrumentos de recogida de información}

Una vez diseñados los instrumentos, distribuimos la muestra en dos grupos (experimental y de comparación) y aplicamos la prueba.

La distribución del alumnado participante en este estudio en los tres niveles de competencia curricular se ha realizado a partir de la aplicación de una prueba pretest de conocimientos matemáticos a un total de 88 de los 90 estudiantes que han compuesto la muestra objeto de estudio. Los resultados obtenidos fueron distribuidos a lo largo de los tres niveles atendiendo a la siguiente estructuración:

- Grupo Bajo: Valor mínimo a Percentil 33.

- Grupo Medio: Percentil 34 a Percentil 66.

- Grupo Alto: Percentil 67 a Valor máximo.

La incorporación de estas categorías a los valores finales obtenidos en dicha prueba de evaluación inicial ha dado como resultado la confección de los tres grupos a partir de los resultados siguientes: grupo básico: 13 a 19; grupo medio: 20 a 25 y grupo avanzado: 26 a 35. 
La distribución de los niveles de competencia curricular en el Centro de Educación Infantil y Primaria Gran Capitán y el número de alumnos y alumnas perteneciente a cada uno de ellos se muestra a continuación:

- Nivel básico: 10 estudiantes.

- Nivel medio: 10 estudiantes.

- Nivel avanzado: 3 estudiantes.

Una vez diseñados los diferentes grupos de nivel competencial, procedimos a distribuir al alumnado en dos grupos de trabajo, un grupo experimental sobre el que se llevaron a cabo los propósitos del estudio (12 estudiantes) y un grupo de comparación que siguió las actividades docentes habituales y que ha servido de referente para advertir la ganancia o pérdida en competencia curricular matemática (11 estudiantes).

\section{4ª Fase. Análisis de los resultados}

Una vez aplicados todos los instrumentos, procedimos a codificar, clasificar y analizar la información recogida con el empleo de diferentes técnicas estadísticas (estudios descriptivos, inferencial y análisis de contenido). Los datos de tipo cuantitativo se han analizados con la ayuda del programa de técnicas estadísticas SPSS y, la información de carácter cualitativa se ha tratado con la técnica del análisis de contenido, técnica de investigación cuya finalidad consiste en la descripción objetiva, sistemática y cuantitativa del contenido de un material determinado, en este caso, de los cuadernos de trabajo del alumnado creados al efecto -nivel básico, medio y avanzado- [(Krippendorf, 2004) y (Neuendorf, 2002)]. Seguidamente, interpretamos los datos obtenidos, establecimos la discusión y conclusiones correspondientes en referencia a las finalidades propuestas.

\section{5a Fase. Difusión de los resultados obtenidos}

Por último, para finalizar este trabajo, hemos elaborado y presentado la memoria de investigación resultante de este proyecto a los respectivos claustros y consejos escolares participantes, así como a la comunidad educativa y científica.

\section{Resultados}

Tras la aplicación del programa de entrenamiento en competencia matemática a través de las diferentes unidades didácticas y aplicada una prueba post-test similar a la implementada al comienzo de este proceso de trabajo, los principales resultados obtenidos, han mostrado un avance significativo en el nivel de competencia curricular en ambos grupos, sin embargo, ha sido el alumnado del grupo experimental quien ha presentado un avance superior al alumnado del grupo de comparación como consecuencia de su participación en las actividades planteadas diferentes a las sesiones ordinarias de aula. Buscando la significación estadística de los datos obtenidos, hemos aplicado un análisis de varianza (n.s.=0.05) (vid. tablas 4 y 5).

Dicha afirmación se ve validada al distribuir la frecuencia de aparición de cada uno de los niveles de competencia curricular en los grupos experimental y de comparación. Podemos advertir, por los datos presentados en la tabla 5, que ha 
habido una disminución del alumnado inicialmente perteneciente al nivel básico en el grupo experimental, así como en el nivel medio, siendo significativamente superior la adscripción de estudiantes, tras su participación en el plan de trabajado diseñado, al grupo de nivel de competencia curricular avanzado. Estos datos muestran un avance significativo en el avance de un nivel competencial inferior a un nivel superior tras la participación en actividades diseñadas para cada grupo de nivel.

Tabla 4. Resultados pretest-postest.

\begin{tabular}{l|l|l|c|c}
\hline $\begin{array}{l}\text { Prueba de } \\
\text { evaluación }\end{array}$ & Grupo & Media & $\begin{array}{c}\text { Desviación } \\
\text { Típica }\end{array}$ & N \\
\hline \multirow{2}{*}{ Pre-test } & Experimental & 21,92 & 5,583 & 12 \\
\cline { 2 - 5 } & De comparación & 21,09 & 3,754 & 11 \\
\hline \multirow{2}{*}{ Post-test } & Experimental & 29,50 & 5,519 & 12 \\
\cline { 2 - 5 } & De comparación & 26,27 & 4,452 & 11 \\
\hline
\end{tabular}

Fuente: PIV-003/08.

Tabla 5. Resultados obtenidos en función del grupo de nivel.

\begin{tabular}{l|c|c}
\hline Grupo de nivel & Prueba de evaluación & $\mathbf{f ~ ( \% )}$ \\
\hline \multirow{2}{*}{ Básico } & Pre-test & $10(43,5 \%)$ \\
\cline { 2 - 3 } & Post-test & $2(8,7 \%)$ \\
\hline \multirow{2}{*}{ Medio } & Pre-test & $10(43,5 \%)$ \\
\cline { 2 - 3 } & Post-test & $3(13,0 \%)$ \\
\cline { 2 - 3 } & Pre-test & $3(13,0 \%)$ \\
\hline
\end{tabular}

Fuente: PIV-003/08.

Estos resultados también se han contrastado con las notas alcanzadas por el alumnado en las evaluaciones realizadas por la tutora, al margen de lo trabajado en las unidades didácticas implementadas por un miembro del equipo de investigación, y siguiendo su correspondiente libro de texto; con relación a la primera unidad didáctica, no se ha podido llevar a cabo la comparación de los resultados, pues se trabajaron contenidos transversales a todas las unidades puestas en marcha por la tutora del grupo clase; sin embargo, los resultados obtenidos por los alumnos y alumnas en las dos unidades didácticas vinculadas con las diseñadas e implementadas para esta investigación han sido los expresados en la tabla 6. 
Tabla 6. Resultados obtenidos por el alumnado en las evaluaciones realizadas por la tutora del grupo de alumnos y alumnas del CEIP Gran Capitán.

\begin{tabular}{|c|c|c|c|c|c|c|c|c|c|c|c|}
\hline \multicolumn{2}{|c|}{ Niveles y grupos } & \multicolumn{5}{|c|}{$\begin{array}{l}\text { Unidad Didáctica: } \\
\text { Longitud }\end{array}$} & \multicolumn{5}{|c|}{$\begin{array}{c}\text { Unidad Didáctica: } \\
\text { Geometría }\end{array}$} \\
\hline Niveles & Grupos & In & Su & $\mathbf{B i}$ & $\mathbf{N}$ & Sb & In & Su & $\mathbf{B i}$ & $\mathbf{N}$ & $\mathrm{Sb}$ \\
\hline \multirow[t]{2}{*}{ Básico } & G. de comparación & 3 & 2 & & & & & 3 & 2 & & \\
\hline & G. experimental & 2 & 1 & 2 & & & & & 3 & 2 & \\
\hline \multirow[t]{2}{*}{ Medio } & G. de comparación & & 3 & 1 & 1 & & & & 2 & 3 & \\
\hline & G. experimental & & 2 & 2 & 1 & & & & 1 & 3 & 1 \\
\hline \multirow{2}{*}{$\begin{array}{l}\text { Avan- } \\
\text { zado }\end{array}$} & G. de comparación & & & & 1 & & & & & & 1 \\
\hline & G. experimental & & & & 2 & 1 & & & & & 3 \\
\hline
\end{tabular}

In: Insuficiente; Su: Suficiente; Bi: Bien; N: Notable; Sb: Sobresaliente.

Fuente: Elaboración propia.

\section{Discusión DE LOS RESULtAdos}

Como consecuencia de los resultados anteriores podemos afirmar, por tanto, que esta metodología de trabajo, el diseño de unidades didácticas atendiendo a las características del alumnado, distribuyéndolos en grupos de nivel de competencia curricular, ha permitido aumentar el nivel competencial de los y las estudiantes de cuarto de educación primaria en el área de Matemáticas. El uso de la Web 2.0, especialmente el vídeo digital, muestra como ha contribuido a dicho incremento tanto en el grupo experimental como en el grupo de comparación, como complemento a la metodología basada en grupos de nivel; de forma concreta, se puede apreciar en los resultados de las unidades didácticas evaluadas por la tutora y de forma más detallada en la tercera unidad, en la que ningún alumno o alumna suspendió (vid. tabla 6).

Los objetivos que nos planteamos al principio de esta investigación, creemos que han sido alcanzados gracias a los instrumentos que se han diseñado e implementado, en este sentido podemos señalar:

Respecto al primer objetivo, aumentar el nivel de competencia curricular del alumnado de cuarto de Educación Primaria en el área de Matemáticas, los resultados anteriores avalan dicha consecución y se demuestra en los avances experimentados por los alumnos y alumnas del grupo experimental. El incremento de los niveles competenciales se ha evidenciado no sólo en cuanto a los referentes de las tres unidades didácticas implementadas, sino también respecto a todo el currículum del área de Matemáticas para el cuarto curso, pues las fichas técnicas que conformaron el pretest-postest abordaban contenidos de todos los bloques y núcleos temáticos de dicho nivel educativo.

En cuanto al segundo objetivo, atender a la diversidad de capacidades, intereses y niveles curriculares del alumnado de un grupo-clase concreto y desarrollar una metodología de trabajo en el aula a través del establecimiento de grupos de nivel diferenciados (básico, medio y avanzado), creemos que ambos se han logrado al 
diseñar los cuadernos de trabajo del alumnado adaptados a los distintos niveles curriculares existentes en nuestras aulas (básico, medio y avanzado) y demostrar su efectividad en los resultados del postest.

En lo que concierne a la hipótesis inicial de esta investigación podemos realizar la siguiente conclusión: El alumnado al que se le aplica el programa formativo (grupo experimental) alcanza unos mejores rendimientos académicos en el área de Matemáticas que el alumnado perteneciente al grupo de comparación. La hipótesis se cumple tal y como se aprecia en la ganancia obtenida por el alumnado del grupo experimental puesta de manifiesto en la tabla 5 .

No obstante, a pesar de estas conclusiones también hemos de destacar la dificultad que entraña la aplicación de este tipo de metodología, pues requiere una coordinación máxima entre todo el profesorado, así como que los centros educativos cuenten en su plantilla con profesorado que posibilite llevar a cabo los desdobles y los consiguientes agrupamientos flexibles. Asimismo, hemos detectado la dificultad de encontrar páginas web o material digital en los que se trabajen de forma nivelada diferentes contenidos educativos para atender a la diversidad curricular del alumnado. Todos estos aspectos exigen grandes esfuerzos de dedicación y económicos respectivamente, pero si de verdad apostamos por incrementar el rendimiento académico de nuestro alumnado y mejorar la calidad de la educación que se le ofrece, todos los esfuerzos serán pocos y poner esta metodología de trabajo al servicio del profesorado es una opción que se ha de considerar a pesar de las reticencias de algunos sectores educativos hacia la misma.

\section{REFERENCIAS BIBLIOGRÁFICAS}

ARNAL, J.; DEL RINCÓN, D. y LATORRE, A. (1992). La investigación educativa. Fundamentos y metodología. Barcelona: Labor.

BORGES PÉREZ, M. I. (2008). Dinamización matemática. Un árbol de Navidad. Unión. Revista Iberoamericana de Educación Matemática, 16, 215-219.

BRAVO, L. y ARRIETA GALLASTEGUI, J. El método Delphi. Su implementación en una estrategia para la enseñanza de las demostraciones geométricas. Revista Iberoamericana de Educación, 35,3: 1-7 (http://www.rieoei.org/deloslectores/804Bravo.PDF) (Consultado 6 de julio de 2010).

CASTAÑO, C.; MAIZ, I.; PALACIO, G. y VILLAROEL, J. D. (2008). Prácticas educativas en entornos web 2.0. Madrid: Pirámide.

CASTILLO, S. (2008). Propuesta pedagógica basada en el constructivismo para el uso óptimo de las TIC en la enseñanza y el aprendizaje de la matemática. Revista Latinoamericana de Investigación en Matemática Educativa, 11 (2), 171-194.

CEBRIÁN HERREROS, M. (2008). La web 2.0 como red social de comunicación e información. Estudios sobre el Mensaje Periodístico, 14, 345-361.

CEBRIÁN DE LA SERNA, M. (2005) (coord.) Vídeo y educación I: vídeos educativos versus vídeos didácticos. Tecnologías de la Información y la Comunicación para la formación de docentes. Madrid: Pirámide. 
COCCONI, P. G. (2008). Arte y artefactos en la educación matemática. Un recorrido por 3 sitios Web. Unión. Revista Iberoamericana de Educación Matemática, 16, 273-288.

DÍAZ ARIAS, R. (2009). El vídeo en el ciberespacio: usos y lenguaje. Comunicar. Revista Científica Iberoamericana de Comunicación y Educación, 33, 141-148.

ECHEVERRÍA, J. (1999). Los señores del aire: Telépolis y el tercer entorno. Barcelona: Destino.

ECHEVERRÍA, J. (2000). Educación y tecnologías telemáticas. Revista Iberoamericana de Educación, 24; 17-36 (http://www.rieoei.org/rie24f.htm) (Consultado 12 de enero de 2009).

FACELLO, T. C. y OSIO, E. B. (2009). Análisis y conclusiones que surgen de la implementación de un taller de Geometría Dinámica para alumnos de profesorado de Matemática. Unión. Revista Iberoamericana de Educación Matemática, 20, 141-151.

FIGUERAS, O. (2005). Atrapados en la explosión del uso de las tecnologías de la información y comunicación. En A. Maz Machado; B. Gómez Alfonso y M. Torralbo Rodríguez (eds.) Investigación en educación matemática. Noveno Simposio de la Sociedad Española de Educación Matemática. Córdoba: Servicio de Publicaciones de la Universidad de Córdoba y SEIEM, 5-16.

FRANZOLIN, F. et al. (2006). Algunas consideraciones sobre los aspectos pedagógicos de los softwares para la enseñanza de las ciencias. Journal of Science Education, 7 (1). (http://www.darwin.futuro.usp.br/site/pesquisas/Artigos/journal_jan_06.pdf) (Consultado 24 de noviembre de 2009).

GALO SÁNCHEZ, J. R. y CAÑAS ESCAMILLA, J. J. (2007). Análisis de una experimentación constructivista con TIC en el aprendizaje de las matemáticas. En P. Bolea; M. Camacho; P. Flores; B. Gómez; J. Murillo; M.T. González (eds). Investigación en educación matemática. Comunicaciones de los grupos de investigación. X Simposio de la SEIEM, 235-245. (http://www.seiem.es/publicaciones/archivospublicaciones/ comunicacionesgrupos/GruposXSimposio.pdf) (Consultado 06 de julio de 2010).

GODINO, J. et al. (2003). Análisis didáctico de recursos interactivos para la enseñanza de la estadística en la escuela. IASE Satellite Conference on Statistic Education and the Internet. (http://www.ugr.es/ jgodino/doctorado/programa2007.htm) (Consultado 23 de noviembre de 2008).

GODINO, J. et al. (2005). Criterios de diseño y evaluación de situaciones didácticas basadas en el uso de medios informáticos para el estudio de las matemáticas. IX Simposio de la SEIEM, (http://www.ugr.es/ jgodino/doctorado/programa2007.htm) (Consultado 23 de noviembre de 2008).

KRIPPENDORF, K. (2004). Content Analysis: An Introduction to Its Methodology. Thousand Oaks, CA: Sage. $2^{\text {nd }}$ edition.

LANDETA, J. (1999). El método Delphi. Barcelona: Ariel.

MANOVICH, L. (2001). The Language of the New Media. Cambridge (Massachusetts): MIT Press.

MURILLO, J. y MARCOS, G. (2007). Una metodología para potenciar y analizar las competencias geométricas y comunicativas. Investigación en Educación Mate- 
mática XI. Tenerife: Sociedad Española de Investigación en Educación Matemática, pp.157-170.

NAVARRO, M. G. (2009). Los nuevos entornos educativos: desafíos cognitivos para una inteligencia colectiva. Comunicar. Revista Científica Iberoamericana de Comunicación y Educación, 33, 141-148.

NATIONAL COUNCIL OF TEACHERS OF MATHEMATICAS (2000). Principles and Standards for School Mathematics. Reston: Va, NCTM.

NEUENDORF, K. (2002). The Content Analysis Guidebook. Thousand Oaks, CA: Sage. SAN MARTíN, P. (2003). Hipertexto: Seis propuestas para este milenio. Buenos Aires: La Crujía.

VÍLCHEZ QUESADA, E. (2006). Impacto de las Nuevas Tecnologías de la Información y la Comunicación para la Enseñanza de la Matemática en la Educación Superior. Revista digital, Matemática, Educación e Internet, 7 (2), (http://www.cidse.itcr.ac.cr/revistamate/ContribucionesV7_n2_2006/IMPACTO/ ImpactoTecn.html) (Consultado 6 de julio de 2010). 